\title{
Emotional Autonomy and Problem Behavior Among Chinese Adolescents
}

\author{
KEE-LEE CHOU \\ Sau Po Centre on Aging \\ University of Hong Kong
}

\begin{abstract}
The author examined the association between emotional autonomy and problem behavior among Chinese adolescents living in Hong Kong. The respondents were 512 adolescents, 16 to 18 years of age, who were interviewed for a cross-sectional study. Three dimensions of emotional autonomy including individuation, nondependency on parents, and de-idealization of parents were significantly and positively correlated with the amount of problem behavior the participants engaged in during the past 6 months. Using a simple linear multiple regression model, the author found that problem behavior was associated with only one aspect of emotional autonomy - individuation. Results indicated that the relationship between problem behavior and three aspects of emotional autonomy was similar in both individualistic and collectivistic societies.
\end{abstract}

Key words: autonomy, Chinese adolescents, problem behavior

EMOTIONAL AUTONOMY has been defined in a test used by Steinberg and Silverberg (1986) as involving three dimensions; (a) de-idealization of parents, or the realization that parents are limited and not all-powerful; (b) nondependency on parents, or the tendency of adolescents not to rely on their parents when they have problems in interpersonal relationship or schools; and (c) individuation, or the belief that their parents do not know or understand them. Presumably, increased emotional autonomy makes a significant contribution to the healthy development of an adolescent (Blos, 1967; Silverberg \& Gondoli, 1996; Steinberg, 1990). While adolescents are gaining greater emotional autonomy, they are maturing and using more of their internal resources and taking respon-

The author thanks his research assistants for their help in the collection of data and those who volunteered to participate in the study. The author also would like to thank the anonymous reviewers for their comments on earlier drafts of this article.

Address correspondence to Kee-Lee Chou, Sau Po Centre on Aging. University of Hong Kong, Pokfulam Road, Hong Kong, China; klchou@hku.hk (e-mail). 
sibility for their actions. Frank, Pirsch, and Wright (1990), who showed that emotional autonomy was positively associated with healthy identity development, supported that position.

Other researchers have argued that there is a positive relationship between emotional autonomy and problem behavior (R. Jessor \& Jessor, 1978). Their rationale is that problem behaviors are linked to several attributes of psychological maturity, such as psychological independence, low parental control, and low parental influence (Hill \& Holmbeck, 1986). In support of that position, Steinberg and Silverberg (1986) showed that emotional autonomy is positively related to susceptibility to peer pressure for problem behavior. Moreover, emotional autonomy was found to be positively associated with problem behaviors such as substance abuse and fighting among adolescents (Turner, Irwin, \& Millstein, 1991).

Emotional autonomy, as one aspect of individual autonomy, has a unique social significance in industrialized Western cultures that emphasize individuality. Previous studies have shown that, although Hong Kong is an advanced industrialized and modernized city, Chinese adolescents living in Hong Kong develop emotional autonomy at a significantly later age than do young people in Western countries (Feldman \& Quatman, 1988; Feldman \& Rosenthal, 1991). To the extent that individual autonomy varies as a developmental imperative among cultural groups, the association between emotional autonomy and problem behavior might also differ.

Recent studies have shown that American and Chinese adolescents display similar levels and types of problem behaviors (C. Chen, Greenberger, Lester, Dong, \& Guo, 1998; Weine, Philips, \& Achenbach, 1995). However, the relationship between problem behavior and emotional autonomy among Chinese adolescents has not been thoroughly studied. In one investigation, Chou (2000b) showed that intimacy with a best friend (especially loyalty and trust in the best friend) was negatively related to problem behavior. Presumably high intimacy with one's friends is related to high emotional autonomy from one's parent. Thus, Chou (2000b) suggested that emotional autonomy might be negatively related to problem behavior. However, Chou (2000a) also showed that emotional autonomy was positively associated with depression. A question remains as to the relationship between emotional autonomy and problem behavior in Chinese youth.

Given those findings, as well as the possibility that the relationship between emotional autonomy and problem behavior might vary across cultures, my purpose in this research was to determine whether emotional autonomy is related to problem behavior among Hong Kong adolescents, and more specifically, whether certain aspects of emotional autonomy are more important than others. To this end, I examined the relationship between problem behavior and three measures of emotional autonomy-individualization, nondependency, and de-idealization. 


\section{Method}

\section{Participants}

The 512 participants in this study were 11 th- and 12 th-grade high school students who participated in the survey in the 1998-1999 school year. Ten schools were randomly selected to participate and one class of 11 th or 12 th graders in each school was randomly selected. Approximately 50 students were selected from each school. The school response rate was $67 \%$, and the participation rate for students was approximately $81 \%$. Of the 544 questionnaires collected, 25 had missing data (more than $20 \%$ missing responses) and 7 appeared to be responded to randomly (for criteria, see Farrell, Danish, \& Howard, 1991). That resulted in a sample size of 512 .

Among the respondents, $238(46.5 \%)$ were boys and $274(53.5 \%)$ were girls. Approximately $85 \%$ came from the 16 - to 18 -year-old age group ( $M=16.9$ years, $S D=1.1$ years). The respondents who had attained 11 th and 12 th grade were $60.5 \%$, and $39.5 \%$, respectively. All of the respondents were high school students, and approximately $7.2 \%$ of them were living in a single-parent household. Data on parental education, measured on a 5-point scale ( $1=$ no formal education to $5=$ university level education $)$ indicated a mean of $2.6(S D=1.1)$ for fathers and a mean of $2.3(S D=1.0)$ for mothers. The most frequently reported monthly household income category, measured in US dollars, was \$1,282-\$1,923 $(54.5 \%)$, followed by the categories of $\$ 641-\$ 1,281(13.9 \%)$ and $\$ 1,924-\$ 2,564$ (13.5\%).

\section{Procedure}

The research staff administered the survey to participating students in the selected classes. The questionnaire took approximately $35 \mathrm{~min}$ to administer. Active personal consents and passive parental consents to participate in this study were sought and obtained for all respondents. To increase the validity of the responses, we made efforts to protect students' privacy; students answered the survey and returned the completed questionnaires in sealed envelopes.

\section{Measures}

Emotional autonomy. The Emotional Autonomy Scale (Steinberg \& Silverberg, 1986) used to assess emotional autonomy is a 13-item scale measuring three aspects of emotional autonomy-individuation, nondependency on parents, and de-idealization of parents. Steinberg and Silverberg have reported excellent psychometric properties (including reliability and validity) of that scale, and the Chinese version of that scale has been used in my previous study (Chou, 2000a). 
The Cronbach's alpha for the three subscales of the Emotional Autonomy Scale for this sample were $.68, .71$, and .64 , respectively.

Problem behavior. I measured problem behavior (R. Jessor, Bos, Vanderryn, Costa, \& Turbin, 1995; Wills, McNamara, Vaccaro, \& Hirky, 1996) by using a 19-item self-report scale that assessed the frequency of 19 types of problem behavior in the past 6 months $(\alpha=89)$. Examples of those listed problem behaviors include fighting, cigarette smoking, shoplifting, vandalizing, lying, and drinking alcoholic beverages. Students were asked to indicate how frequently they engaged in each of 19 listed problem behaviors during the past 6 months, using a 5-point scale ranging from 0 (never) to 5 (five times or more). I summed the 19 items to produce a problem-behavior score in which higher scores indicated more frequent problem behavior.

\section{Statistical Analysis}

First, I examined the bivariate correlations between the independent variables (age, gender $[1=$ boy, $2=$ girl $]$, marital status of parents $[1=$ divorced, $2=$ married $]$, education level of parents $[1=$ elementary or below to $5=$ university or above], monthly household income measured in US dollars [ $1=\$ 640$ or below; $2=\$ 641$ to $\$ 1,281 ; 3=\$ 1,282$ to $\$ 1,923 ; 4=\$ 1,924$ to $\$ 2,564 ; 5=\$ 2,565$ to $\$ 3,205 ; 6=\$ 3,206$ to $\$ 3,846 ; 7=\$ 3,847$ or above $]$ ) and the dependent variables (individuation, nondependency on parents, and de-idealization of parents, and problem behavior). One simple linear multiple regression analysis was performed, and I entered the predictive variables, which included age, gender, education, marital status of parents, education of father and mother, household income, and the three measurements of emotional autonomy into the model by the forced-entry method. The dependent variable for the regression model was problem behavior.

\section{Results}

\section{Correlations Between Problem Behavior and the Independent Variables}

The intercorrelations among all independent and dependent variables for all participants are shown in Table 1. I found positive and significant correlations between problem behavior and the three subscales of emotional autonomy-individuation, nondependency on parents, and de-idealization of parents. No significant association was found between the three subscales of emotional autonomy and the sociodemographic characteristics of young people in this sample, with one exception: Nondependency on parents was significantly and negatively related to maternal education. The strength of the association between all independent variables being used in the multiple regression analysis was moderate $(.61 \geq|r| \geq .01)$. 


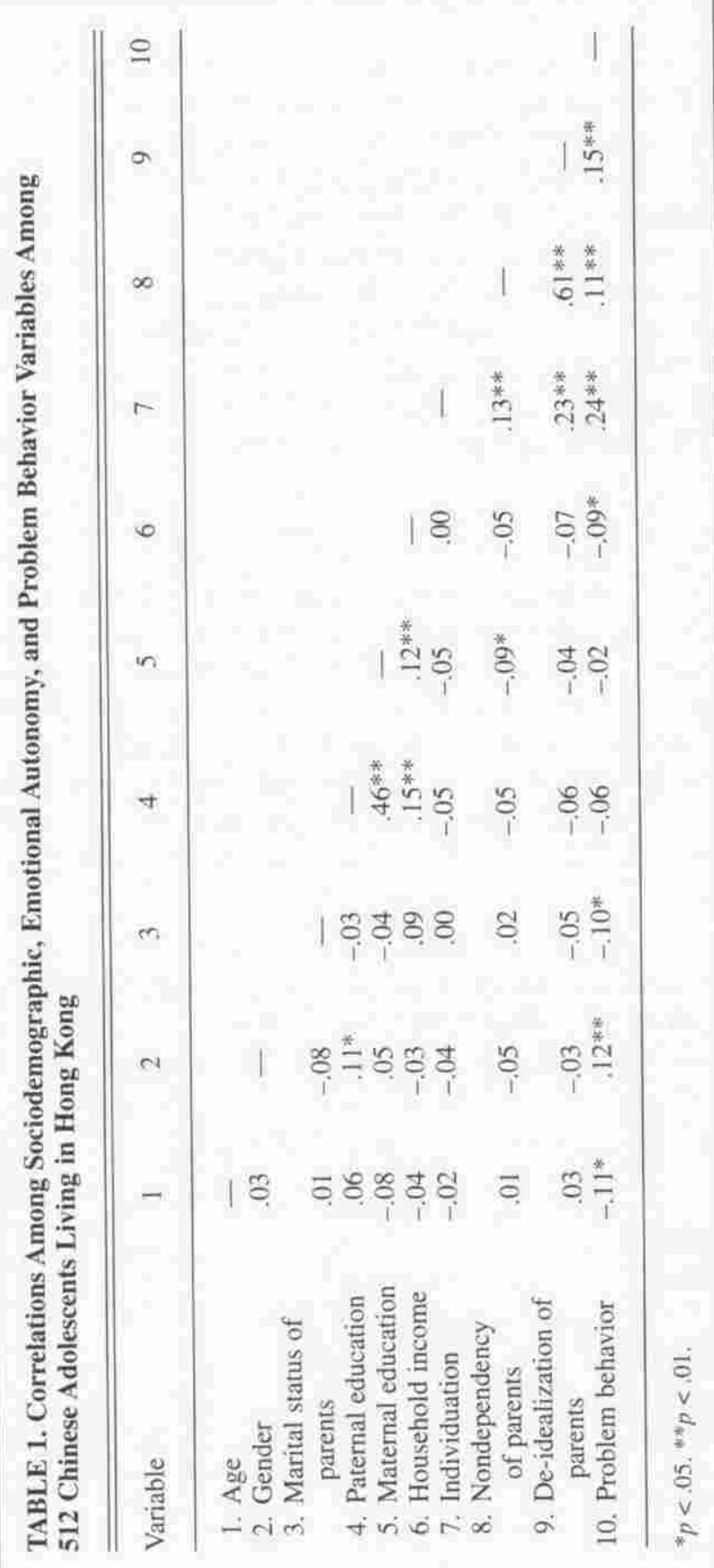




\section{Relationship Between Problem Behavior and Independent Variables}

Table 2 shows that problem behavior scores were significantly related to age, gender, marital status of parent, monthly household income, and individuation. Specifically, being younger, being female, having divorced parents, having a higher monthly household income, or having a higher level of individuation were associated with more frequent engagement in problem behavior. The regression analysis model accounted for $11 \%$ of the variance in intimacy. $F(9,502)=7.06, p<.001$, $R^{2}=.11$. I found moderate correlations between the predictive variables used in the regression model; therefore, tolerance values of predictive variables were obtained. All of the tolerance values were at least .59 or higher, which is greater than the common cutoff threshold of . I (Hair, Anderson, Tatham, \& Black, 1995). As a result, the multicollinearity between predictor variables was at an acceptable level.

\section{Discussion}

In the present investigation, I examined the association between different aspects of emotional autonomy and problem behavior. Two important findings in this sample of Chinese adolescents living in Hong Kong should be emphasized. First, the results of multiple regression analysis indicated that problem behavior is positively associated with individuation. That result replicates previous findings that individuation is positively related to problem behavior in American adolescents (Z. Y. Chen \& Dornbusch, 1998). Those findings suggest that as part of the separation-individuation process in adolescence, there is likely to be a major

\section{TABLE 2. Summary of Multiple Regression Analysis for Variables Predicting Adolescent Problem Behavior Scores}

\begin{tabular}{lccc}
\hline \hline Variable & $B$ & SE B & $\beta$ \\
\hline Age & -.03 & .01 & $-.10^{*}$ \\
Gender & -.07 & .03 & $-.10^{*}$ \\
Marital status of parents & -.16 & .06 & $-.12^{* *}$ \\
Paternal education & -.02 & .02 & -.05 \\
Maternal education & .00 & .02 & .00 \\
Household income & .03 & .01 & $-.11^{*}$ \\
Individuation & .17 & .04 & $.21^{* *}$ \\
Nondependency of parents & .02 & .04 & .03 \\
Deidealization of parents & .07 & .05 & .09 \\
\hline
\end{tabular}

Note. $F(9,502)=7.06^{=1 *}: R^{2}=.11$.

${ }^{*} p<.05 .{ }^{* *} p<.01 .{ }^{* * *} p<.001$. 
reorganization of intimate interpersonal relationships, with decreasing dependence on family and increasing closeness to peers (Petersen, 1988). However, to explore the impact of that process on misconduct in adolescence, one must investigate why emotional autonomy leads to a higher level of problem behavior.

Second, although significant bivariate correlations between problem behavior and all measures of emotional autonomy (including individuation, nondependency on parents, and de-idealization of parents) were found, the effect of nondependency on parents and de-idealization of parents disappeared in the multivariate model that included other dimensions of emotional autonomy. Those findings illustrate that it is crucial to study the effects of different aspects of emotional autonomy on problem behavior among adolescents in a multivariate model.

However, one should use caution in interpreting the findings of the current study because some significant correlation coefficients found between problem behavior and some independent variables used in the regression model were quite small. To be conservative, those correlations should be viewed as having theoretical, rather than practical, significance.

\section{Conclusion}

The findings of the current study suggest that individuation of emotional autonomy is positively associated with problem behavior in Chinese adolescents living in Hong Kong. That means that if adolescents feel that their parents do not know or understand them, they are likely to engage in problem behavior. Therefore, communication between adolescents and their parents is very important so that they will know and understand each other to a greater extent.

\section{REFERENCES}

Blos, P. (1967). The second individuation process. Psychoanalytic Study of the Child, 22. 162-186.

Chen, C., Greenberger, E., Lester, J., Dong, Q., \& Guo, M. S. (1998). A cross-cultural study of family and peer correlates of adolescent misconduct. Developmental Psychology, 34, 770-781.

Chen, Z. Y., \& Dornbusch, S. M. (1998). Relating aspects of adolescent emotional autonomy to academic achievement and deviant behavior. Journal of Adolescent Research, 13, 293-319.

Chou, K. L. (2000a). Emotional autonomy and depression among Chinese adolescents. The Journal of Genetic Psychology. 161, 161-168.

Chou, K. L. (2000b). Intimacy and psychosocial adjustment in Chinese adolescents. The Journal of Genetic Psychology, 161, 141-151.

Farrell, A. D., Danish, S. J., \& Howard, C. W. (1991). Evaluation of data screening methods in surveys of adolescents' drug use. Psychological Assessment: A Journal of Consulting and Clinical Psychology, 3, 295-298.

Feldman, S. S., \& Quatman, T. (1988). Factors influencing age expectations for adolescent autonomy: A study of early adolescents and parents. Journal of Early Adolescence, 8, 325-343. 
Feldman, S. S., \& Rosenthal, D. A. (1991). Age expectations of behavioral autonomy in Hong Kong. Australian and American youth: The influence of family variables and adolescents' values. International Journal of Psychology, 26, 1-23.

Frank, S., Pirsch, L, \& Wright, V. (1990). Late adolescents' perceptions of their relationships with their parents: Relationships among deidealization, autonomy, relatedness, and insecurity and implications for adolescent adjustment and ego identity status. Journal of Youth and Adolescence, 19.571-588.

Hair, J. F., Anderson, R. E., Tatham, R. L., \& Black, W. C. (1995). Multivariate data analysis (4th ed.). Englewood Cliffs, NJ: Prentice Hall.

Hill, J. P., \& Holmbeck, G. N. (1986). Attachment and autonomy during adolescence. Annals of Child Development, 3, 145-189.

Jessor, R., Bos, J. V. D., Vanderryn, J., Costa, F. M., \& Turbin, M. S. (1995). Protective factors in adolescent problem behavior: Moderator effects and development change. Developmental Psychology, 31, 923-933.

Jessor, R., \& Jessor, S. L. (1978). Theory testing in longitudinal research on marijuana use, In D. B. Kandel (Ed.), Longitudinal research on drug use: Empirical findings and methodological issues (pp. 41-72). Washington, DC: Hemisphere.

Petersen, A. C. (1988). Adolescent development. Anmual Review of Psychology, 39. 583-607.

Silverberg, S. B. \& Gondoli, D. M. (1996). Autonomy in adolescence: A contextualized perspective. In G. R. Adams. R. Montemayor. \& T. P. Gullotta (Eds.), Psychosocial development during adolescence (pp. 12-61). London: Sage.

Steinberg. L. (1990). Autonomy, conflict, and harmony in the family relationship. In S. Feldman \& G. Elliott (Eds.), At the threshold: The developing adolescent (pp. 255-276). Cambridge, MA: Harvard University Press.

Steinberg, L., \& Silverberg, S. B. (1986). The vicissitudes of autonomy in early adolescence. Child Development, 57, 841-851.

Turner, R., Irwin, C., \& Millstein, S. (1991). Family structure, family processes, and experimenting with substances during adolescence. Journal of Research on Adolescence, 1. 93-106.

Weine, J. R., Philips, J. S., \& Achenbach. T. M. (1995). Behavioral and emotional problems among Chinese and American children: Parent and teacher reports for ages 6 to 13. Journal of Abnormal Child Psychology, 23, 619-639.

Wills, T. A., McNamara, G., Vaccaro, D., \& Hirky, A. E. (1996). Escalated substance use: A longitudinal grouping analysis from early to middle adolescence. Journal of Abnormal Psychology, 105, 166-180. 